\title{
IN BRIEF
}

\section{$\Rightarrow$ METABOLISM}

\section{PFC neurons take control of food intake}

The prefrontal cortex (PFC) regulates behaviours, but its role in controlling food intake specifically is not well understood. The authors showed that in mice, dopamine receptor 1 (D1R)-expressing PFC neurons are activated during feeding. Optogenetic stimulation of D1R-expressing PFC neurons in the left hemisphere increased food intake, whereas bilateral inhibition reduced it. D1R-expressing neuron stimulation also selectively increased activity in the medial basolateral amygdala (mBLA) - one of the target areas of these neurons - and stimulation of the terminals of D1R-expressing PFC neurons in the $\mathrm{mBLA}$ increased food intake. These data point to a prefrontal-mBLA circuit for the control of food intake.

ORIGINAL RESEARCH PAPER Land, B. B. et al. Medial prefrontal D1 dopamine neurons control food intake. Nature Neurosci. http://dx.doi.org/10.1038/nn.3625 (2014)

\section{$\Rightarrow$ NEUROANATOMY}

\section{Following subcortical development}

Structural MRI studies have provided insight into cerebral cortex development in humans, but little is known about the development of subcortical structures. This longitudinal study measured the shape and volume of cortical and subcortical regions in MRI scans of males and females obtained between the ages of 5 and 25 years. In both sexes, the pallidum reached its peak volume first, followed by the cortex, striatum and thalamus, although there was local heterogeneity in volume change within each subcortical structure. The authors also noted sex differences in the timing of volume change in the structures.

ORIGINAL RESEARCH PAPER Raznahan, A. et al. Longitudinal four-dimensional mapping of subcortical anatomy in human development. Proc. Natl Acad. Sci. USA http://dx.doi. org/10.1073/pnas.1316911111 (2014)

\section{$\Rightarrow$ SPATIAL PROCESSING}

\section{The place of head-direction cells}

In rats, spatial navigation involves hippocampal place cells, which encode the animal's location in an environment, and head-direction cells, which are located outside the hippocampus and encode the orientation of the animal's head. This study showed that in bats exploring an open-field arena, many hippocampal neurons encode both location and head direction. Moreover, in some neurons, the head-direction tuning was preserved even when the animal was outside the neuron's place field. Further analysis may reveal the existence of similar place-head-direction cells in rats. Alternatively, there may be species differences in neural encoding of head direction.

ORIGINAL RESEARCH PAPER Rubin, A., Yartsev, M. M. \& Ulanovsky, N. Encoding of head direction by hippocampal place cells in bats. J. Neurosci. 34, 1067-1080 (2014)

\section{$\Rightarrow$ SYNAPTIC PHYSIOLOGY}

\section{mROS link metabolism to GABA transmission}

Cellular metabolism regulates excitatory neurotransmission, but what is its role in GABAergic neurotransmission? The authors show that disrupting mitochondrial function with antimycin A increases GABAergic transmission at synapses onto cerebellar stellate cells. This effect was mediated by antimycin A-induced generation of mitochondrial-derived reactive oxygen species (mROS) acting postsynaptically to recruit extrasynaptic $\alpha 3$-subunit-containing $\mathrm{GABA}_{A}$ receptors. This finding points to a role for ROS as signalling molecules.

ORIGINAL RESEARCH PAPER Accardi, M. V. et al. Mitochondrial reactive oxygen species regulate the strength of inhibitory GABA-mediated synaptic transmission. Nature Commun. 5, 3168 (2014) 